\title{
Optimal Distributed Controller Design for Nonlinear Coupled Dynamical Networks
}

\author{
Hao Zhang, ${ }^{1}$ Huaicheng Yan, ${ }^{2}$ and Qijun Chen ${ }^{1}$ \\ ${ }^{1}$ Department of Control Science and Engineering, Tongji University, Shanghai 200092, China \\ ${ }^{2}$ The Key Laboratory of Advanced Control and Optimization for Chemical Processes of Ministry of Education, \\ School of Information Science and Engineering, East China University of Science and Technology, Shanghai 200237, China
}

Correspondence should be addressed to Huaicheng Yan; hcyan@ecust.edu.cn

Received 6 January 2014; Accepted 6 February 2014; Published 11 March 2014

Academic Editor: Xudong Zhao

Copyright (C) 2014 Hao Zhang et al. This is an open access article distributed under the Creative Commons Attribution License, which permits unrestricted use, distribution, and reproduction in any medium, provided the original work is properly cited.

\begin{abstract}
This paper is concerned with the optimal distributed impulsive controller design for globally exponential synchronization of nonlinear dynamical networks with coupling delay. By the Lyapunov-Razumikhin method, a novel criterion is proposed to guarantee the global exponential synchronization of the coupled delayed network with distributed impulsive control in terms of matrix inequalities. The sum of coupling strengths of the distributed impulsive control is minimized to save the control effort. Finally, the effectiveness of the proposed method has been demonstrated by some simulations.
\end{abstract}

\section{Introduction}

During the last decade, a great amount of effort has been devoted to the study of synchronization of coupled networks due to their applications in many fields including secure communication, neural networks [1], and information science [210]. It is well known that there are three primary methods to be used to study the synchronization of the coupled network, that is, the Master Stability Function method, Lyapunov's direct method, and Connection Graph Stability method. Based on these methods, many research results have been reported on the synchronization from different points of view. Bounded $H_{\infty}$ synchronization and state estimation for discrete time-varying stochastic complex networks over a finite horizon have been investigated in [2]. A timevarying complex dynamical network model and its controlled synchronization criteria have been considered in [5]. In [6], pinning synchronization of directed and undirected complex dynamical networks has been studied. An adaptive feedback controller has been proposed in [3] to synchronize a general complex dynamical network with delayed nodes.

In recent years, there are many control schemes that have been introduced to realize the network synchronization. For example, robust impulsive synchronization [11, 12], pinning control [13], and adaptive control [14]. In impulsive control, the impulse signal is input into the nodes only at impulsive instance. The essential benefit of the impulsive control approach is derived from the fact that such control requires much less information, computational power, and bandwidth in sensing data communications in coupled networks and increases the robustness against the disturbance. Hence, the impulsive control is more effective compared with the control approaches using continuous measurement. In [13, 15-17], impulsive controllers for the stabilization or synchronization of complex dynamical networks are applied. Considering the impulsive effect as disturbance of the system, many researchers have done investigation to analyze the bound of the impulsive interval, parameter of the node, and topology of the network [11, 18-22]. In these references, the impulsive controller or disturbance only effect on the local sensor or node and the coupling of the impulsive effect has not been considered. However, in this paper, the distributed impulsive controller is designed to synchronize the coupled network not only based on its local measurement but also on its neighboring measurement according to the topology of distributed impulsive controller. Although the synchronization of coupled networks has been investigated intensively in recent years, however, to our best knowledge, the 
distributed impulsive control for nonlinear coupled networks has received little attention, in particular the optimal of the coupling strength. This motivates the current study.

In this paper, we aim to deal with the synchronization of nonlinear coupled networks with time-varying delay via distributed impulsive control and specify the minimum coupling strengths of the corresponding links in the distributed controller topology. The rest of the paper is organized as follows. In Section 2, the problem we are considering is described and some useful lemmas and definitions are presented. Section 3 provides the main results of this paper. The distributed impulsive controller is designed to synchronize the coupled networks with time-varying delay and the sum of the coupling strength of the controller is minimum. An example is presented in Section 4 and some simulations are presented to illustrate the effectiveness of the proposed methods. Finally, the conclusions are given in Section 5.

Notations. For vector $x=\operatorname{col}\left(x_{1}, \ldots, x_{n}\right) \in \mathbb{R}^{n}$ and matrix $A=\left(a_{i j}\right)_{n \times n} \in \mathbb{R}^{n \times n},\|x\|$ and $\|A\|$ denote 2 -norms of $x$ and $A$, respectively. A real matrix $P>0(P<0)$ denotes $P$ being a positive (negative) definite matrix, and $A>B$ means $A-B>0 . M^{\top}$ denotes the transpose of matrix $M$. The identity matrix of order $m$ is denoted as $I_{m}$ (or simply $I$ if no confusion arises). Moreover, matrices are assumed to have compatible dimensions if not explicitly stated. $*$ denotes the symmetric block of a symmetric matrix, and diag $\{\cdot\}$ denotes the block diagonal matrix. $\lambda_{\max }(P)$ and $\lambda_{\min }(P)$ are used to denote the maximum and minimum eigenvalue of matrix $P$, respectively. $\mathbb{N}$ is the set of all positive natural numbers.

\section{Preliminaries and Problem Formulation}

We consider a dynamical network consisting of $N$ linearly coupled oscillators. Each node of the network is an ndimensional dynamical system, which is described by

$$
\begin{array}{r}
\dot{x}_{i}(t)=f\left(x_{i}(t)\right)+\sum_{j=1}^{N} a_{i j} \Gamma x_{j}(t-\tau(t))+u_{i}(t), \\
i=1,2, \ldots, N,
\end{array}
$$

where $x_{i}(t)=\left(x_{i 1}(t), x_{i 2}(t), \ldots, x_{i n}(t)\right)^{\top} \in \mathbb{R}^{n}$ is the state of the $i$ th oscillator, $f\left(x_{i}(t)\right) \in \mathbb{R}^{n} \rightarrow \mathbb{R}^{n}$ is a continuous function, and there exists a unique continuous solution for any initial condition. Suppose that the uniform Lipschitz condition holds; that is, for any $x, y \in \mathbb{R}^{n}$. Then, there exists a positive constant $L>0$ such that $\|f(x)-f(y)\| \leq L \| x-$ $y \| . \Gamma \in \mathbb{R}^{n \times n}$ is the inner-coupling matrix, and $a_{i j}$ is the coupling strength from the $j$ th oscillator to the $i$ th oscillator, where $a_{i j}>0$ means that the $i$ th oscillator is coupled with the $j$ th oscillator directly and $a_{i j}=0$ means that the $i$ th oscillator is decoupled from the $j$ th oscillator. Suppose that the communication topology is strongly connected [23]. $\tau(t)$ is the time delay between nodes which satisfies $\tau(t) \leq \tau$ $[13,24-26]$. Moreover, $u_{i}(t) \in \mathbb{R}^{n}$ are the controllers designed for the network.

The topology of the coupled networks in (1) is described as a directed graph $\mathscr{G}=(\mathscr{V}, \mathscr{E})$ of order $N$ with the set of nodes $\mathscr{V}=\{1,2, \ldots, N\}$, and the elements in $\mathscr{V}$ are the oscillator's indexes. $\mathscr{E}$ is a set of directed link sets, and when oscillator $i$ is coupled with oscillator $j$ directly, there is a directed link $(i, j) \in \mathscr{E}$ from node $j$ to node $i$; that is, $\mathscr{E}=\left\{(i, j) \mid a_{i j}>0, \forall i, j=1,2, \ldots, N\right\}$. The set of neighbors of vertex $i$ is denoted by $\mathcal{N}_{i}=\{j \in \mathscr{V}:(i, j) \in \mathscr{E}\}$. In the following, we introduce some definitions and lemma that are essential for the development of main results in this paper.

Definition 1. The coupled network (1) is said to be globally exponentially synchronous if there exist constants $\gamma>0$ and $\mu>0$, such that for any initial conditions the inequality

$$
\left\|x_{i}(t)-x_{j}(t)\right\|^{2} \leq \gamma e^{-\mu t}
$$

holds for $t \geq 0$.

Definition 2. A coupling matrix $\mathscr{A}$ is defined as

$$
\begin{aligned}
\mathscr{A} & =\left[\begin{array}{cccc}
-\sum_{j=2}^{N} a_{1 j} & a_{12} & \cdots & a_{1 N} \\
a_{21} & -\sum_{j=1, j \neq 2}^{N} a_{2 j} & \cdots & a_{2 N} \\
\vdots & \ddots & \ddots & \ddots \\
a_{N 1} & a_{N 2} & \cdots & -\sum_{j=1}^{N-1} a_{N j}
\end{array}\right] \\
& =\sum_{(i, j) \in \mathscr{C}} a_{i j} A_{i j},
\end{aligned}
$$

where $A_{i j} \in \mathbb{R}^{N \times N}$ denotes the constant matrices corresponding to the directed links $(i, j)$ and each $A_{i j}$ only contains two nonzero entries, that is, -1 at the iith entry and 1 at the $i j$ th entry. From Definition 2, the coupled network (1) can be rewritten in a compact form:

$$
\dot{\bar{x}}(t)=f(\bar{x}(t))+\mathscr{A} \otimes \Gamma \bar{x}(t-\tau(t))+u(t),
$$

where $\bar{x}(t)=\left(x_{1}^{\top}(t), x_{2}^{\top}(t), \ldots, x_{N}^{\top}(t)\right)^{\top}, f(\bar{x}(t))=\left(f\left(x_{1}^{\top}(t)\right)\right.$, $\left.f\left(x_{2}^{\top}(t)\right), \ldots, f\left(x_{N}^{\top}(t)\right)\right)^{\top}$, and $u(t)=\left[u_{1}^{\top}(t), u_{2}^{\top}(t), \ldots\right.$, $\left.u_{N}^{\top}(t)\right]^{\top}$. Define matrices $G$ and $W$ as

$$
\begin{aligned}
G & =\left[\begin{array}{ccccc}
1 & -1 & 0 & \cdots & 0 \\
0 & \ddots & \ddots & \ddots & 0 \\
\vdots & \ddots & 1 & -1 & \vdots \\
0 & \cdots & 0 & 1 & -1
\end{array}\right] \in \mathbb{R}^{N-1 \times N}, \\
W & =\left[\begin{array}{cccc}
1 & 1 & 1 & 1 \\
0 & 1 & \cdots & 1 \\
0 & 0 & \ddots & \vdots \\
0 & 0 & \cdots & 1 \\
0 & 0 & \cdots & 0
\end{array}\right] \in \mathbb{R}^{N \times N-1},
\end{aligned}
$$

which satisfies

$$
G W=I_{N-1}, \quad \mathscr{A}=\mathscr{A} W G, \quad G \otimes I_{m}\left[\begin{array}{c}
x_{1} \\
\vdots \\
x_{N}
\end{array}\right]=\left[\begin{array}{c}
e_{1} \\
\vdots \\
e_{N-1}
\end{array}\right],
$$


where $e_{i}=x_{i}-x_{i+1}, i=1, \ldots, N-1$. By the multiplication of $G \otimes I_{m}$ on (4), one has

$$
\dot{\bar{e}}(t)=F(\bar{x}(t))+((G \mathscr{A} W) \otimes \Gamma) \bar{e}(t-\tau(t))+\bar{u}(t),
$$

where

$$
\begin{gathered}
\bar{e}(t)=\left[\begin{array}{c}
\dot{e}_{1}(t) \\
\vdots \\
\dot{e}_{N-1}(t)
\end{array}\right], \\
F(\bar{x}(t))=\left[\begin{array}{c}
f\left(x_{1}\right)-f\left(x_{2}\right) \\
\vdots \\
f\left(x_{N-1}\right)-f\left(x_{N}\right)
\end{array}\right], \\
\bar{e}(t-\tau(t))=\left[\begin{array}{c}
e_{1}(t-\tau(t)) \\
\vdots \\
e_{N-1}(t-\tau(t))
\end{array}\right], \\
\bar{u}(t)=\left[\begin{array}{c}
u_{1}(t)-u_{2}(t) \\
\vdots \\
u_{N-1}(t)-u_{N}(t)
\end{array}\right] .
\end{gathered}
$$

In order to achieve synchronization of coupled network (1) and inspired by the controller proposed in [27], a distributed impulsive controller for the $i$ th node is designed as

$$
\begin{array}{r}
u_{i}(t)=\sum_{k=1}^{\infty} \sum_{j \in \mathcal{N}_{i}} v_{i j} \Gamma\left(x_{j}(t)-x_{i}(t)\right) \delta\left(t-t_{k}\right), \\
i=1,2, \ldots, N,
\end{array}
$$

where $v_{i j}$ denotes the coupling strength corresponding to the directed links $(i, j)$, which need to be designed. The impulsive instant sequence $\left\{t_{k}\right\}_{0}^{\infty}$ satisfies $0 \leq t_{0}<t_{1}<t_{2}<\cdots<t_{k}<$ $\cdots, \lim _{k \rightarrow \infty} t_{k}=\infty . \delta(\cdot)$ is the Dirac impulsive function; that is, $\delta(t)=0$ for $t \neq 0$, and $\int_{-\infty}^{\infty} \delta(t) d t=1$. In many applications, the Dirac delta function is usually used to model an impulse. Taking integral on both sides of (1) from $t_{k}-h$ to $t_{k}+h$, where $h \rightarrow 0^{+}$, one has

$$
\Delta x_{i}\left(t_{k}\right)=\sum_{j \in \mathcal{N}_{i}} v_{i j} \Gamma\left(x_{j}\left(t_{k}\right)-x_{i}\left(t_{k}\right)\right),
$$

where $\Delta x_{i}\left(t_{k}\right)=x_{i}\left(t_{k}^{+}\right)-x_{i}\left(t_{k}^{-}\right), \lim _{h \rightarrow 0^{+}} x_{i}\left(t_{k}-h\right)=x_{i}\left(t_{k}^{-}\right)$, and $\lim _{h \rightarrow 0^{+}} x_{i}\left(t_{k}+h\right)=x_{i}\left(t_{k}^{+}\right)$, with discontinuity instants $0 \leq t_{0}<t_{1}<t_{2}<\cdots<t_{k}<\cdots$. Without loss of generality, it is assumed that $x_{i}\left(t_{k}\right)=x_{i}\left(t_{k}^{-}\right)=\lim _{h \rightarrow 0^{+}} x_{i}\left(t_{k}-h\right)$; that is, $x_{i}(t)$ is left continuous at $t=t_{k}$. Equation (10) implies that the node $i$ will suddenly update its state variables not only according to the state variables of itself but also its neighbors at instant $t_{k}$.

Since the distributed controller can use the original topology of the coupled network and will not cause additional effort in building new links, the topology of the distributed controller is designed as the same with the coupled network
(1). Hence, the coupling matrix of the distributed impulsive controller can be defined as

$$
\begin{aligned}
\mathscr{V} & =\left[\begin{array}{cccc}
-\sum_{j=2}^{N} v_{1 j} & v_{12} & \cdots & v_{1 N} \\
v_{21} & -\sum_{j=1, j \neq 2}^{N} v_{2 j} & \cdots & v_{2 N} \\
\vdots & \ddots & \ddots & \ddots \\
v_{N 1} & v_{N 2} & \cdots & -\sum_{j=1}^{N-1} v_{N j}
\end{array}\right] \\
& =\sum_{(i, j) \in \mathscr{E}} v_{i j} A_{i j},
\end{aligned}
$$

where $A_{i j} \in \mathbb{R}^{N \times N}$ is defined in (3). Then, one can rewrite (10) into a compact form:

$$
\Delta \bar{x}\left(t_{k}\right)=(\mathscr{V} \otimes \Gamma) \bar{x}\left(t_{k}\right),
$$

where $\Delta \bar{x}^{\top}\left(t_{k}\right)=\left(\Delta x_{1}\left(t_{k}\right), \Delta x_{2}\left(t_{k}\right), \ldots, \Delta x_{N}\left(t_{k}\right)\right)^{\top}, \bar{x}^{\top}\left(t_{k}\right)=$ $\left(x_{1}\left(t_{k}\right), x_{2}\left(t_{k}\right), \ldots, x_{N}\left(t_{k}\right)\right)^{\top}$. By the multiplication of $G \otimes I_{m}$ on (12), one has

$$
\Delta \bar{e}\left(t_{k}\right)=((G \mathscr{V} W) \otimes \Gamma) \bar{e}\left(t_{k}\right),
$$

where $\Delta \bar{e}\left(t_{k}\right)=\left(\Delta e_{1}^{\top}\left(t_{k}\right), \Delta e_{2}^{\top}\left(t_{k}\right), \ldots, \Delta e_{N-1}^{\top}\left(t_{k}\right)\right)^{\top}, \bar{e}\left(t_{k}\right)=$ $\left(e_{1}^{\top}\left(t_{k}\right), e_{2}^{\top}\left(t_{k}\right), \ldots, e_{N-1}^{\top}\left(t_{k}\right)\right)^{\top}, \Delta e_{i}\left(t_{k}\right)=\Delta x_{i}\left(t_{k}\right)-\Delta x_{i+1}\left(t_{k}\right)$, and $e_{i}\left(t_{k}\right)=x_{i}\left(t_{k}\right)-x_{i+1}\left(t_{k}\right), i=1,2, \ldots, N-1$.

From (7) and (13), the impulsively controlled error system can be described by the following impulsive differential equation:

$$
\begin{gathered}
\dot{\bar{e}}(t)=F(\bar{x}(t))+((G \mathscr{A} W) \otimes \Gamma) \bar{e}(t-\tau(t)), \quad t \neq t_{k}, \\
\Delta \bar{e}\left(t_{k}\right)=((G \mathscr{V} W) \otimes \Gamma) \bar{e}\left(t_{k}\right), \\
\bar{e}\left(t_{0}+\theta\right)=\phi(\theta), \quad-\tau \leq \theta \leq 0,
\end{gathered}
$$

where $\phi \in \mathscr{C}\left([-\tau, 0], \mathbb{R}^{n}\right)$.

Lemma 3 (see [28]). For matrices $A, B, C$, and $D$ with appropriate dimensions, one has

$$
\begin{gathered}
(A \otimes B)^{\top}=A^{\top} \otimes B^{\top}, \\
A \otimes(B+C)=A \otimes B+A \otimes C, \\
(A \otimes B)(C \otimes D)=A C \otimes B D, \\
(A \otimes B)^{-1}=A^{-1} \otimes B^{-1} .
\end{gathered}
$$

In the following section, the exponential synchronization of coupled networks with distributed impulsive control is investigated based on this model.

\section{Main Results}

In this section, a novel contradiction method is proposed to analyze the global exponential synchronization of system (1) to obtain an optimal distributed impulsive control law. 
Theorem 4. Let $\rho=\sup _{k \in \mathbb{N}}\left\{t_{k}-t_{k-1}\right\}<\infty$, given positive scalars $\alpha$, and $0<\gamma<1$, if there exists matrix $P>0$, matrix $\mathscr{V}$, and positive scalar $\varepsilon$, such that the following inequalities

$$
\begin{gathered}
{\left[\begin{array}{ccc}
\varepsilon \bar{L}+\left(\frac{\alpha}{\gamma}+\frac{\ln \gamma}{\rho}\right) P & P((G \mathscr{A} W) \otimes \Gamma) & P \\
* & -\alpha P & 0 \\
* & * & -\varepsilon I
\end{array}\right]<0,} \\
{\left[\begin{array}{cc}
-\gamma P & (I+(G \mathscr{V} W) \otimes \Gamma)^{\top} P \\
* & -P
\end{array}\right]<0,}
\end{gathered}
$$

hold; then the coupled network (1) can achieve global exponential synchronization.

Proof. In the following, for convenience, we assume $t_{0}=0$. For $\phi \in \mathscr{C}\left([-\tau, 0], \mathbb{R}^{n}\right)$, we denote the solution $\bar{e}\left(t, t_{0}, \phi\right)$ of (14) by $\bar{e}(t)$. Consider a Lyapunov function candidate for the error dynamic system (14) as

$$
V(t)=\bar{e}^{\top}(t) P \bar{e}(t),
$$

where $P>0$. Calculating the Dini derivative of $V(t)$ along the solution of the system (14) for $t \in\left(t_{k-1}, t_{k}\right], k \in \mathbb{N}$, one has

$$
\begin{aligned}
D V(t)= & 2 \bar{e}^{\top}(t) P(F(\bar{x}(t))+((G \mathscr{A} W) \otimes \Gamma) \bar{e}(t-\tau(t))) \\
\leq & \bar{e}^{\top}(t)\left(\frac{1}{\varepsilon} P^{2}+\varepsilon \bar{L}\right) \bar{e}(t) \\
& +2 \bar{e}^{\top}(t) P((G \mathscr{A} W) \otimes \Gamma) \bar{e}(t-\tau(t)),
\end{aligned}
$$

where $\bar{L}=I_{N-1} \otimes L^{2}$. Noting that $0<\gamma<1$ and inequality (16), there exist positive scalars $h$, $\mu$, and $\varepsilon$, where $h+\gamma<1$, such that the following linear matrix inequality is satisfied:

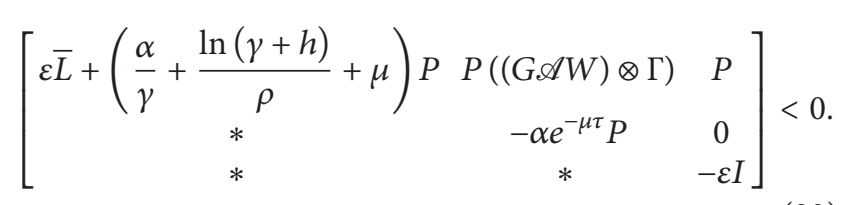

From (17), one has

$$
(I+(G \mathscr{V} W) \otimes \Gamma)^{\top} P(I+(G \mathscr{V} W) \otimes \Gamma)-\gamma P<0 .
$$

Hence,

$$
\begin{aligned}
V\left(t_{k}^{+}\right)= & \bar{e}^{\top}\left(t_{k}^{+}\right) P \bar{e}\left(t_{k}^{+}\right) \\
= & \bar{e}^{\top}\left(t_{k}\right)(I+(G \mathscr{V} W) \otimes \Gamma)^{\top} \\
& \times P(I+(G \mathscr{V} W) \otimes \Gamma) \bar{e}\left(t_{k}\right) \\
\leq & \gamma \bar{e}^{\top}\left(t_{k}\right) P \bar{e}\left(t_{k}\right)=\gamma V\left(t_{k}\right) .
\end{aligned}
$$

Let $\mathscr{W}(t)=e^{\mu t} V(t)$; then

$$
\dot{\mathscr{V}}(t)=\mu e^{\mu t} V(t)+e^{\mu t} V(t), \quad t \in\left[t_{k-1}, t_{k}\right), k \in \mathbb{N} .
$$

Let $v>\lambda_{\max }(P) / \gamma \lambda_{\min }(P)$ be a fixed number. In the following, we will prove that $\mathscr{W}(t)<\lambda_{\text {min }}(P) \nu\|\phi\|^{2}, t \geq t_{0}-\tau$. Note that

$$
\begin{aligned}
\mathscr{W}\left(t_{0}+\theta\right) & \leq \lambda_{\max }(P)\|\phi\|^{2}<\gamma \lambda_{\min }(P) \nu\|\phi\|^{2} \\
& <\lambda_{\min }(P) \nu\|\phi\|^{2}
\end{aligned}
$$

for $-\tau \leq \theta \leq 0$. We will first prove that

$$
\mathscr{W}(t)<\lambda_{\min }(P) \nu\|\phi\|^{2}, \quad \text { for } t \in\left(t_{0}, t_{1}\right),
$$

via contradiction. Suppose this is not the case; that is, there exists a $t \in\left(t_{0}, t_{1}\right)$ such that $\mathscr{W}(t) \geq \lambda_{\min }(P) v\|\phi\|^{2}$. Set $t^{*}=$ $\inf \left\{t \in\left(t_{0}, t_{1}\right): \mathscr{W}(t) \geq \lambda_{\text {min }}(P) \nu\|\phi\|^{2}\right\}$; then one has

$$
\mathscr{W}\left(t^{*}\right)=\lambda_{\min }(P) v\|\phi\|^{2}, \quad t^{*} \in\left(t_{0}, t_{1}\right) .
$$

Noticing (24), one has $\mathscr{W}\left(t_{0}\right)<\gamma \lambda_{\min }(P) \nu\|\phi\|^{2}$. Set $\bar{t}=$ $\sup \left\{t \in\left(t_{0}, t^{*}\right): \mathscr{W}(t) \leq \gamma \lambda_{\min }(P) \nu\|\phi\|^{2}\right\}$; then one has

$$
\begin{array}{r}
\mathscr{W}(\bar{t})=\gamma \lambda_{\min }(P) \nu\|\phi\|^{2}, \quad \bar{t} \in\left(t_{0}, t^{*}\right), \\
\mathscr{W}(t) \geq \gamma \lambda_{\min }(P) \nu\|\phi\|^{2} \geq \gamma \mathscr{W}(t+\theta), \\
\theta \in[-\tau, 0] \text { for } t \in\left(\bar{t}, t^{*}\right) .
\end{array}
$$

Hence, one has that, for $t \in\left(\bar{t}, t^{*}\right)$,

$\dot{\mathscr{W}}(t)$

$$
\begin{aligned}
& \leq \mu e^{\mu t} V(t)+e^{\mu t} \dot{V}(t) \\
& +\left(\frac{\alpha}{\gamma} \mathscr{W}(t)-\alpha \mathscr{W}(t-\tau(t))\right) \\
& \leq e^{\mu t}\left(\bar{e}^{\top}(t) \mu P \bar{e}(t)\right) \\
& +e^{\mu t}\left(\bar{e}^{\top}(t)\left(\frac{1}{\varepsilon} P^{2}+\varepsilon \bar{L}\right) \bar{e}(t)+2 \bar{e}^{-\top}(t)\right. \\
& \quad \times P((G \mathscr{A} W) \otimes \Gamma) \bar{e}(t-\tau(t)))
\end{aligned}
$$

$$
\begin{aligned}
& +e^{\mu t}\left(\frac{\alpha}{\gamma} \bar{e}^{\top}(t) P e(t)-\alpha e^{-\mu \tau} \bar{e}^{\top}(t-\tau(t)) P e(t-\tau(t))\right) \\
\leq & e^{\mu t} \xi^{\top}(t)
\end{aligned}
$$

$$
\begin{aligned}
& \times\left[\begin{array}{cc}
\frac{1}{\varepsilon} P^{2}+\varepsilon \bar{L}+\left(\frac{\alpha}{\gamma}+\frac{\ln (\gamma+h)}{\rho}+\mu\right) P & P((G \mathscr{A} W) \otimes \Gamma) \\
* & -\alpha e^{-\mu \tau} P
\end{array}\right] \\
& \times \xi(t)+\gamma_{1} \mathscr{W}(t),
\end{aligned}
$$

where $\gamma_{1}=-\ln (\gamma+h) / \rho, \xi^{\top}(t)=\left[\bar{e}^{\top}(t), \bar{e}^{\top}(t-\tau(t))\right]$. Noticing (20), one has

$$
\dot{\mathscr{W}}(t) \leq \gamma_{1} \mathscr{W}(t), \quad t \in\left[\bar{t}, t^{*}\right]
$$


then

$$
\mathscr{W}\left(t^{*}\right) \leq \mathscr{W}(\bar{t}) e^{\gamma_{1}\left(t^{*}-\bar{t}\right)}<\gamma \lambda_{\min }(P) \nu\|\phi\|^{2} e^{\gamma_{1} \rho}
$$

the last inequality is derived by using (27) and $\bar{t}-t^{*}<\rho$. Since $\gamma e^{\gamma_{1} \rho}<1$, inequality (31) becomes

$$
\mathscr{W}\left(t^{*}\right)<\lambda_{\min }(P) \nu\|\phi\|^{2},
$$

which contradict to (26). Now we have proved that inequality (25) holds. Next, we assume that

$$
\mathscr{W}(t)<\lambda_{\min }(P) \nu\|\phi\|^{2}, \quad \text { for } t \in\left[t_{0}-\tau, t_{m}\right], m \in \mathbb{N} .
$$

We want to prove that

$$
\mathscr{W}(t)<\lambda_{\min }(P) \nu\|\phi\|^{2}, \quad \text { for } t \in\left(t_{m}, t_{m+1}\right] .
$$

Suppose this is not the case; that is, there exists a $t \in\left(t_{m}, t_{m+1}\right]$ such that

$$
\mathscr{W}(t) \geq \lambda_{\min }(P) \nu\|\phi\|^{2}
$$

Set $t^{*}=\inf \left\{t \in\left(t_{m}, t_{m+1}\right]: \mathscr{W}(t) \geq \lambda_{\text {min }}(P) v\|\phi\|^{2}\right\}$; then

$$
\mathscr{W}\left(t^{*}\right)=\lambda_{\min }(P) \nu\|\phi\|^{2} .
$$

From (22) and (33), we have

$$
\mathscr{W}\left(t_{m}^{+}\right) \leq \gamma \mathscr{W}\left(t_{m}\right)<\gamma \lambda_{\min }(P) \nu\|\phi\|^{2}
$$

Set $\bar{t}=\sup \left\{t \in\left(t_{m}, t^{*}\right]: \mathscr{W}(t) \leq \gamma \lambda_{\min }(P) \nu\|\phi\|^{2}\right.$; then $\mathscr{W}(\bar{t})=$ $\gamma \lambda_{\text {min }}(P) \nu\|\phi\|^{2}$, and $\mathscr{W}(t) \geq \gamma \lambda_{\text {min }}(P) \nu\|\phi\|^{2} \geq \gamma \mathscr{W}(t+\theta)$ for $t \in\left[\bar{t}, t^{*}\right]$. One has

$$
\begin{aligned}
& \dot{\mathscr{W}}(t) \\
& \leq \mu e^{\mu t} V(t)+e^{\mu t} \dot{V}(t)+\left(\frac{\alpha}{\gamma} \mathscr{W}(t)-\alpha \mathscr{W}(t-\tau(t))\right) \\
& \leq e^{\mu t} \xi^{\top}(t) \\
& \times\left[\begin{array}{cc}
\frac{1}{\varepsilon} P^{2}+\varepsilon \bar{L}+\left(\frac{\alpha}{\gamma}+\frac{\ln (\gamma+h)}{\rho}+\mu\right) P & P((G \mathscr{A} W) \otimes \Gamma) \\
* & -\alpha e^{-\mu \tau} P
\end{array}\right] \\
& \times \xi(t)+\gamma_{1} \mathscr{W}(t) \\
& \leq \gamma_{1} \mathscr{W}(t) \text {, }
\end{aligned}
$$

for $t \in\left[\bar{t}, t^{*}\right]$. Hence,

$$
\begin{aligned}
\mathscr{W}\left(t^{*}\right) & \leq \mathscr{W}(\bar{t}) e^{\gamma_{1}\left(t^{*}-\bar{t}\right)} \leq \gamma \lambda_{\min }(P) \nu\|\phi\|^{2} e^{\gamma_{1} \rho} \\
& <\lambda_{\min }(P) \nu\|\phi\|^{2}
\end{aligned}
$$

which yields a contradiction to (36). Therefore, inequality (34) holds. By mathematical induction we have

$$
\mathscr{W}(t)<\lambda_{\min }(P) \nu\|\phi\|^{2}, \quad \text { for } t \geq t_{0} .
$$

On the other hand, note that $V(t) \geq \lambda_{\min }(P)\|\bar{e}(t)\|^{2}$; one has

$$
\left\|\bar{e}\left(t, t_{0}, \phi\right)\right\|^{2}<v\|\phi\|^{2} e^{-\mu\left(t-t_{0}\right)}
$$

thus from Definition 1, the coupled network (1) is globally synchronized under the distributed controller (9).

Remark 5. Theorem 4 outlines the principle of determining the distributed controller gains. However, it does not provide an optimal coupling strength. Next, the sum of all coupling strengths is minimized by solving the following optimisation problem:

$$
\min _{P, v_{i j}} \sum_{(i, j) \in \mathscr{E}} v_{i j}
$$

subject to (16), (17).

Remark 6. When the coupled network (1) is given, then the coupling strength $a_{i j}$ is known; consequently, $A_{i j}$ is also known. The topology of the distributed controller can be designed as the same with coupled network (1). Only the coupling strength $v_{i j}$ needs to be determined. Our aim is to optimize the coupling strength of the controller and make the sum of all coupling strengths $\sum_{(i, j) \in \mathscr{E}} v_{i j}$ being minimized. Since $P$ and $\mathscr{V}$ are unknown matrices, inequality (17) is not linear matrix inequality and cannot be solved efficiently. We will propose the following algorithm to find a suboptimal solution of the $v_{i j}$.

Step 1. Let the positive matrix $P=I$, and let $\beta$ be a small positive scalar.

Step 2. Solve the linear matrix inequality (17); one will have $v_{i j}$.

Step 3. Using the value of $v_{i j}$ obtained by Step 2, take a positive matrix $P$ that is to be determined. Solving the optimization problem as follows:

$$
\begin{aligned}
& \max _{P} \delta \\
& {\left[\begin{array}{ccc}
\varepsilon \bar{L}+\left(\frac{\alpha}{\gamma}+\frac{\ln \gamma}{\rho}\right) P & P((G \mathscr{A} W) \otimes \Gamma) & P \\
* & -\alpha P & 0 \\
* & 0 & -\varepsilon I
\end{array}\right]<0,} \\
& l=1,2, \ldots, r, \\
& {\left[\begin{array}{cc}
-\gamma P & (I+(G \mathscr{V} W) \otimes \Gamma)^{\top} P \\
* & -P
\end{array}\right]<-\delta I,}
\end{aligned}
$$

where $\delta$ is positive scalar.

Step 4. If $\delta>\beta$, then go back to Step 2; else, terminate the algorithm.

Thus we can obtain the coupling strength $v_{i j}$ with the smallest coupling sum $\sum_{(i, j) \in \mathscr{E}} v_{i j}$ by this iterative algorithm. 


\section{Illustrative Example}

In this section, a numerical example of global exponential synchronization of a coupled network with 5 Lorenz oscillators is provided to illustrate the design procedure of the proposed distributed impulsive controller. The functions of Lorenz oscillators are given by

$$
f\left(x_{i}\right)=\left[\begin{array}{c}
-10 x_{i 1}+10 x_{i 2} \\
28 x_{i 1}-x_{i 2}-x_{i 1} x_{i 3} \\
x_{i 1} x_{i 2}-\frac{1}{3} x_{i 3}
\end{array}\right], \quad i=1,2,3,4,5,
$$

with $x_{i}=\left[\begin{array}{lll}x_{i 1} & x_{i 2} & x_{i 3}\end{array}\right]^{\top}$. Let $L=10$; the inner-coupling matrix is given by $\Gamma=I$ and the coupling delay is set as $0.1 \sin (t)$. The coupling matrix of the given coupled network is given by

$$
\mathscr{A}=0.1 *\left[\begin{array}{ccccc}
-1 & 0 & 1 & 0 & 0 \\
0 & -1 & 0 & 1 & 0 \\
0 & 1 & -1 & 0 & 0 \\
0 & 1 & 0 & -1 & 0 \\
0 & 0 & 1 & 0 & -1
\end{array}\right]
$$

then the coupling matrix of distributed controller can be designed as follows:

$$
\mathscr{V}=\left[\begin{array}{ccccc}
-v_{13} & 0 & v_{13} & 0 & 0 \\
0 & -v_{24} & 0 & v_{24} & 0 \\
0 & v_{32} & -v_{32} & 0 & 0 \\
0 & v_{42} & 0 & -v_{42} & 0 \\
0 & 0 & v_{53} & 0 & -v_{53}
\end{array}\right],
$$

such that the sum of coupling strengths $\sum_{(i, j) \in \mathscr{E}} v_{i j}$ is as small as possible. Let $\alpha=1, \rho=0.01$, and $\gamma=0.7$. Solving matrix inequalities (16) and (17) by the method proposed in Remark 5, we have $\min \sum_{(i, j) \in \mathscr{E}} v_{i j}=1.98$. The states of the coupled network without control and with distributed impulsive control are shown in Figures 1 and 2, respectively. From Figure 2, we find that the coupled network (1) is globally exponentially synchronous under the distributed controller.

\section{Conclusion}

In this paper, we have studied the distributed impulsive controller design for globally exponential synchronization of nonlinear networks with coupling delay. The sum of coupling strengths of the distributed impulsive control is minimized to save the control effort. Some criteria have been derived based on Lyapunov-Razumikhin method to guarantee the global exponential synchronization of the coupled network with distributed impulsive control in terms of matrix inequalities. A numerical example has been presented to demonstrate the usefulness and effectiveness of the proposed approach. Further research directions would include the investigation on more general nonlinear systems.

\section{Conflict of Interests}

The authors declare that there is no conflict of interests regarding the publication of this paper.

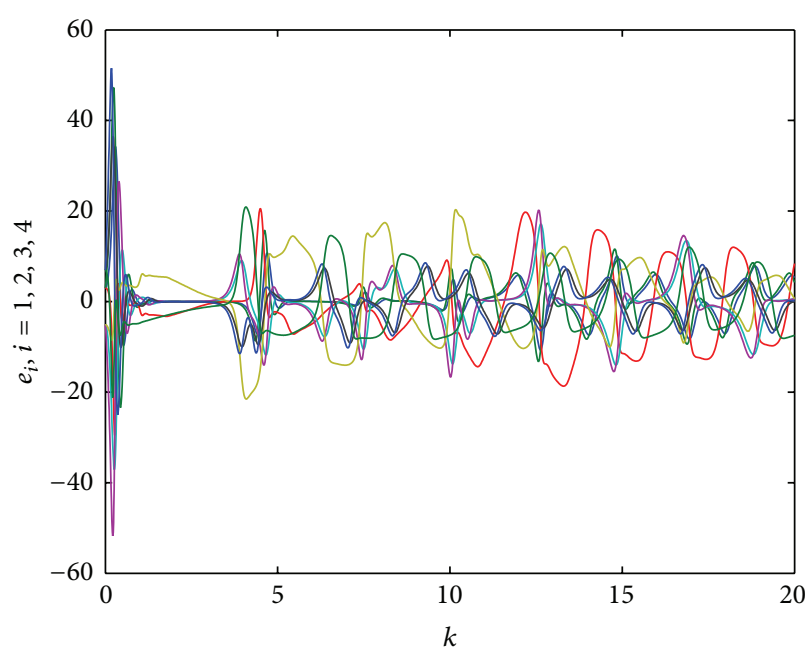

FIGURE 1: The error states of the coupled network without control.

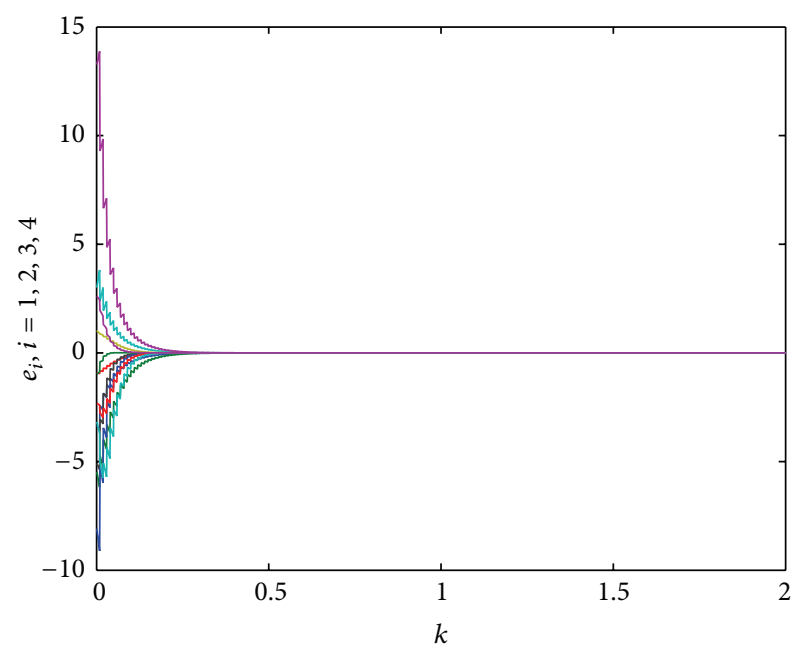

FIgURE 2: The error states of the coupled network with distributed impulsive control.

\section{Acknowledgments}

This work is supported by the National Natural Science Foundation of China (61273026, 61004028, 61272064, and 91120308), the Innovation Program of Shanghai Municipal Education Commission (12zz052), and the Fundamental Research Funds for the Central Universities.

\section{References}

[1] H. Li, H. Gao, and P. Shi, "New passivity analysis for neural networks with discrete and distributed delays," IEEE Transactions on Neural Networks, vol. 21, no. 11, pp. 1842-1847, 2010.

[2] B. Shen, Z. Wang, and X. Liu, "Bounded $H_{\infty}$ synchronization and state estimation for discrete time-varying stochastic complex networks over a finite horizon," IEEE Transactions on Neural Networks, vol. 22, no. 1, pp. 145-157, 2011.

[3] Q. J. Zhang, J. A. Lu, J. H. Lu, and C. K. Tse, "Adaptive feedback synchronization of a general complex dynamical network with 
delayed nodes," IEEE Transactions on Circuits and Systems II, vol. 55 , no. 2 , pp. $183-187,2008$.

[4] L. Chen, J. A. Lu, and C. K. Tse, "Synchronization: an obstacle to identification of network topology," IEEE Transactions on Circuits and Systems II, vol. 56, no. 4, pp. 310-314, 2009.

[5] J. H. Lü and G. R. Chen, "A time-varying complex dynamical network model and its controlled synchronization criteria," IEEE Transactions on Automatic Control, vol. 50, no. 6, pp. 841846, 2005.

[6] Q. Song and J. Cao, "On pinning synchronization of directed and undirected complex dynamical networks," IEEE Transactions on Circuits and Systems I, vol. 57, no. 3, pp. 672-680, 2010.

[7] H. Zhang, H. C. Yan, F. W. Yang, and Q. J. Chen, "Quantized control design for impulsive fuzzy networked systems," IEEE Transactions on Fuzzy Systems, vol. 19, no. 6, pp. 1153-1162, 2011.

[8] L. X. Zhang, H. J. Gao, and O. Kaynak, "Network-induced constraints in networked control systems-a survey," IEEE Transactions on Industrial Informatics, vol. 9, no. 1, pp. 403-416, 2013.

[9] H. J. Gao, T. W. Chen, and J. Lam, "A new delay system approach to network-based control," Automatica, vol. 44, no. 1, pp. 39-52, 2008.

[10] H. C. Yan, Z. Z. Su, H. Zhang, and F. W. Yang, "Observerbased $H_{\infty}$ control for discrete-time stochastic systems with quantisation and random communication delays," IET Control Theory \& Applications, vol. 7, no. 3, pp. 372-379, 2013.

[11] H. L. Xu, Y. Q. Chen, and K. L. Teo, "Global exponential stability of impulsive discrete-time neural networks with time-varying delays," Applied Mathematics and Computation, vol. 217, no. 2, pp. 537-544, 2010.

[12] D. Gong, H. Zhang, Z. Wang, and J. Liu, "Synchronization analysis for complex networks with coupling delay based on TS fuzzy theory," Applied Mathematical Modelling, vol. 36, no. 12, pp. 6215-6224, 2012.

[13] J. Zhou, Q. J. Wu, and L. Xiang, "Pinning complex delayed dynamical networks by a single impulsive controller," IEEE Transactions on Circuits and Systems I, vol. 58, no. 12, pp. 28822893, 2011.

[14] F. Sorrentino and E. Ott, "Adaptive synchronization of dynamics on evolving complex networks," Physical Review Letters, vol. 100, no. 11, Article ID 114101, 4 pages, 2008.

[15] Y. Dai, Y. Cai, and X. Xu, "Synchronisation analysis and impulsive control of complex networks with coupling delays," IET Control Theory \& Applications, vol. 3, no. 9, pp. 1167-1174, 2009.

[16] Y. W. Wang, M. Yang, H. O. Wang, and Z. H. Guan, "Robust stabilization of complex switched networks with parametric uncertainties and delays via impulsive control," IEEE Transactions on Circuits and Systems I, vol. 56, no. 9, pp. 2100-2108, 2009.

[17] Q. Zhang, J. A. Lu, and J. Zhao, "Impulsive synchronization of general continuous and discrete-time complex dynamical networks," Communications in Nonlinear Science and Numerical Simulation, vol. 15, no. 4, pp. 1063-1070, 2010.

[18] B. Liu, X. Liu, G. Chen, and H. Wang, "Robust impulsive synchronization of uncertain dynamical networks," IEEE Transactions on Circuits and Systems I, vol. 52, no. 7, pp. 1431-1441, 2005.

[19] X. S. Yang, J. D. Cao, and J. Q. Lu, "Synchronization of delayed complex dynamical networks with impulsive and stochastic effects," Nonlinear Analysis, vol. 12, no. 4, pp. 2252-2266, 2011.
[20] J. Q. Lu, D. W. C. Ho, J. D. Cao, and J. Kurths, "Exponential synchronization of linearly coupled neural networks with impulsive disturbances," IEEE Transactions on Neural Networks, vol. 22, no. 2, pp. 329-335, 2011.

[21] J. Q. Lu, D. W. C. Ho, and J. D. Cao, "A unified synchronization criterion for impulsive dynamical networks," Automatica, vol. 46, no. 7, pp. 1215-1221, 2010.

[22] S. G. Peng, "Delay-independent global exponential stabilization for stochastic fuzzy systems with time delay via impulsive fuzzy control," in Proceedings of the 29th Chinese Control Conference (CCC '10), pp. 1049-1052, July 2010.

[23] C. Wu, "Synchronization in networks of nonlinear dynamical systems coupled via a directed graph," Nonlinearity, vol. 18, no. 3, pp. 1057-1064, 2005.

[24] T. H. Lee, J. H. Park, O. M. Kwon, and S. M. Lee, "Synchronization of a time-varying delayed complex dynamical network with nonidentical nodes and free coupling matrix," in Proceedings of the International Conference on Control, Automation and Systems (ICCAS '11), pp. 1837-1840, October 2011.

[25] R. Cepeda-Gomez and N. Olgac, "An exact method for the stability analysis of linear consensus protocols with time delay," IEEE Transactions on Automatic Control, vol. 56, no. 7, pp. 17341740, 2011.

[26] C. Yoshioka and T. Namerikawa, "Observer-based consensus control strategy for multi-agent system with communication time delay," in Proceedings of the IEEE International Conference on Control Applications (CCA '08), pp. 1037-1042, San Antonio, Tex, USA, September 2008.

[27] Z. H. Guan, Z. W. Liu, G. Feng, and Y. W. Wang, "Synchronization of complex dynamical networks with time-varying delays via impulsive distributed control," IEEE Transactions on Circuits and Systems I, vol. 57, no. 8, pp. 2182-2195, 2010.

[28] L. Huang, Linear Algebra in System and Control Theory, Science Press, Beijing, China, 1984 


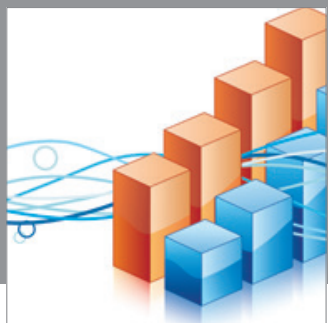

Advances in

Operations Research

mansans

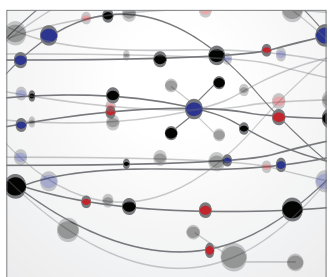

The Scientific World Journal
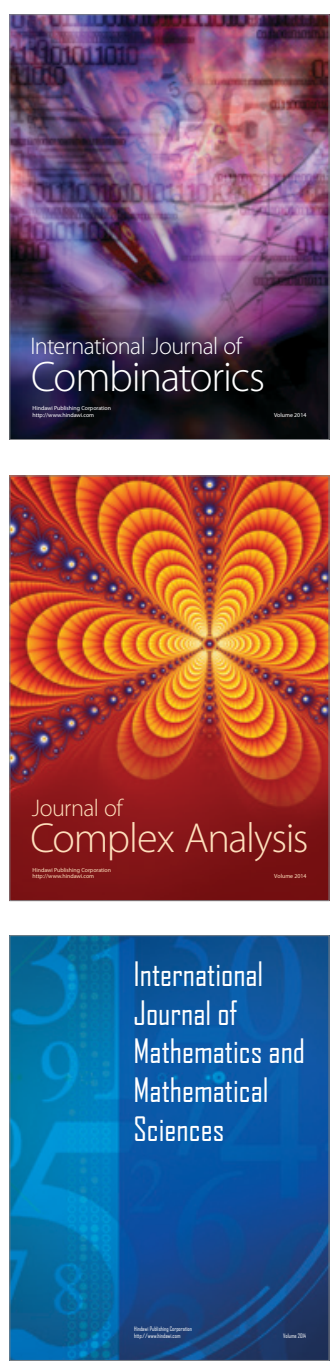
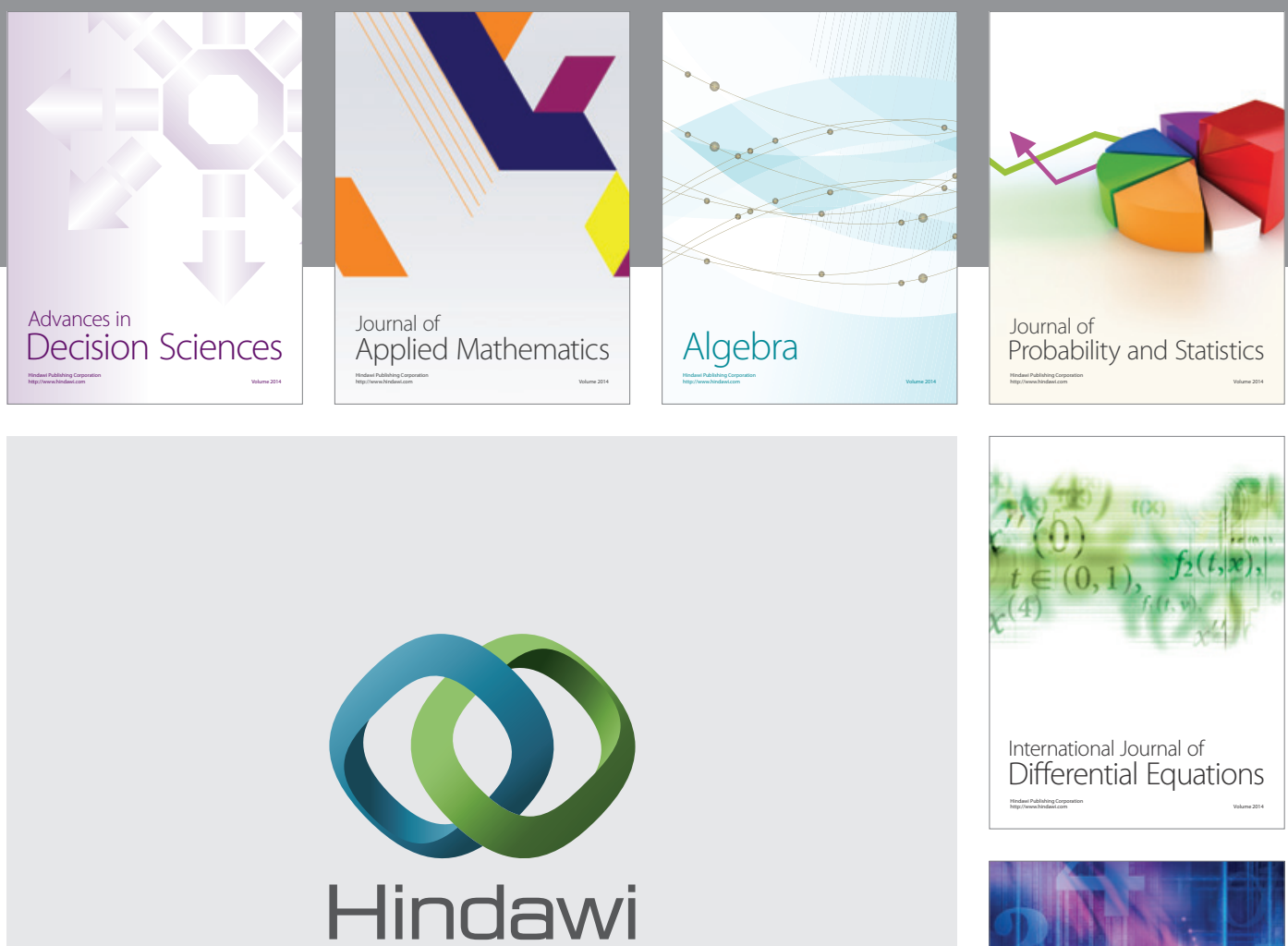

Submit your manuscripts at http://www.hindawi.com
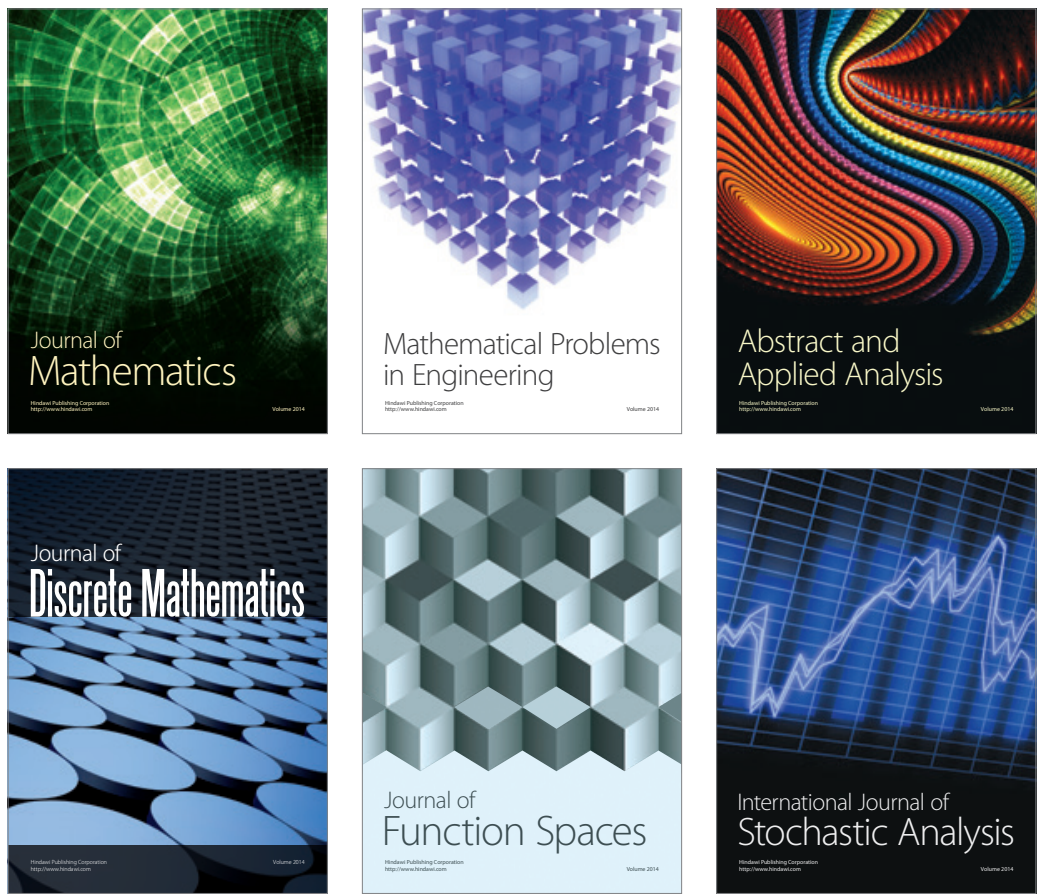

Journal of

Function Spaces

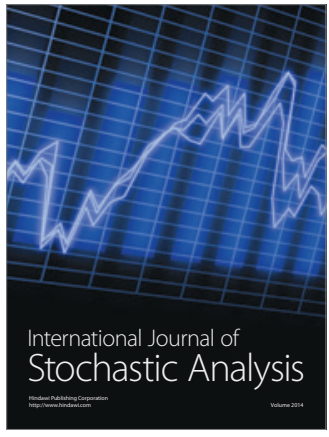

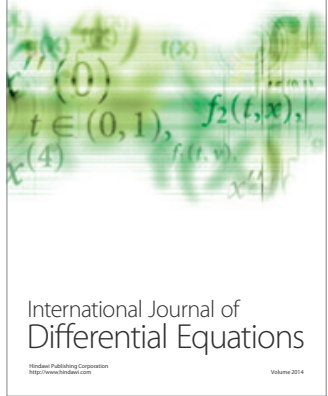
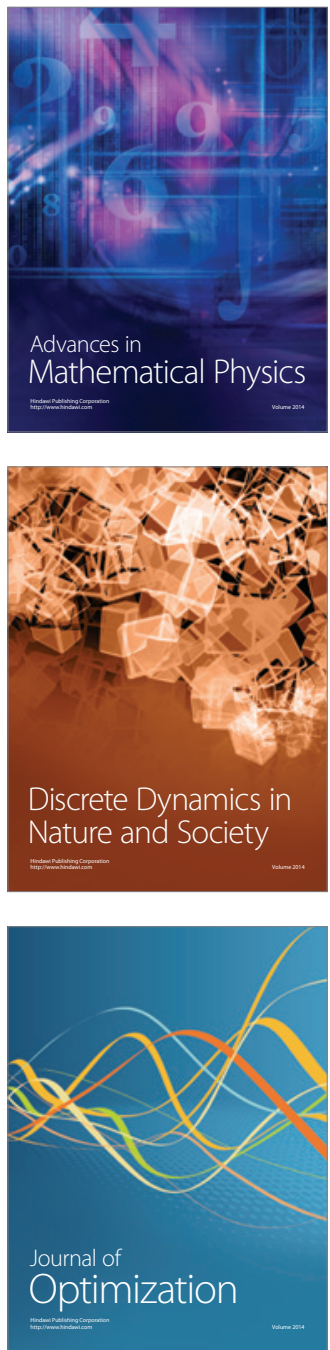